\title{
THE USE OF THE CHEMICAL METHOD FOR THE DETERMINATION OF INTERFACIAL AREAS IN GAS-LIQUID CONTACTORS
}

\author{
M. H. OYEVAAR and K. R. WESTERTERP ${ }^{\dagger}$ \\ Department of Chemical Engineering, Chemical Reaction Engineering Laboratories, University of Twente, \\ PO Box 217, 7500 AE Enschede, Netherlands
}

(Received 24 October 1988; accepted 20 January 1989)

\begin{abstract}
The interfacial area $a_{\text {chem }}$ in a gas-liquid contactor as determined by the chemical method deviates from the true geometrical interfacial area $a_{\text {gea }}$, because the overall conversion of the gas phase reactant represents an incorrect average if bubble sizes and residence times arc not uniform. The deviations of $a_{\mathrm{chem}}$ from $a_{\mathrm{gco}}$ become larger the broader the distribution $\tau_{b} / d_{b}$ and the higher the overall conversion $\boldsymbol{\Omega}_{A}$ of the reactant in the gas phase. Model calculations, which take into account both the effect of gas phase backmixing as well as the effect of bubble coalescence on the deviation of $a_{\mathrm{chcm}}$ from $a_{\mathrm{g} i \mathrm{o}}$, are performed for a mechanically agitated gas-liquid reactor and a bubble column at practical micro- and macromixing conditions. For a gas-liquid model reaction, which is first-order in the gas phase reactant, it is found that: (1) for a mechanically agitated reactor the error in $a_{\text {chem }}$ will always be smaller than $10 \%$ if $\Omega_{A}$ is lower than 0.99 , and (2) for a bubble column the error in $a_{\text {chem }}$ will be smaller than $20 \%$ for most practical applications if $\Omega_{A}$ is lower than 0.99 . Gas-liquid model reaction systems with absorption of $\mathrm{CO}_{2}$ in alkanolamine solutions are recommended for the determination of interfacial areas in gas-liquid contactors.
\end{abstract}

\section{INTRODUCTION}

Since its introduction by Westerterp et al. (1963) the chemical method has been widely used for the determination of mass transfer parameters in gas-liquid contactors. Both the interfacial area and the volumetric gas and liquid phase mass transfer coefficients, which are important parameters for the design of gas-liquid contactors, can be determined by the chemical method. The major drawback of the chemical method is its restriction to specific gas-liquid systems and their particular physico-chemical properties. Therefore, in recent years numerous alternative physical methods have been developed for the determination of interfacial areas.

All these physical methods are based on local determinations of the interfacial area $a$, which have to be integrated in order to obtain the overall value. Some of these physical methods determine the values for the local interfacial areas by means of optical techniques based on light scattering and light reflection [see, for example, Calderbank (1958) and Sridhar and Potter (1978)]. Other methods determine bubble size distributions either by means of photography or with optical and conductivity probes [see Reith and Beek (1968), Deckwer et al. (1978), Buchholtz et al. (1979) and Schumpe and Deckwer (1982)]. The interfacial area is then evaluated from the Sauter mean diameter $d_{s}$ and the gas hold-up $\varepsilon_{G}$ according to

$$
a=\frac{6 \varepsilon_{G}}{d_{s}} \text {. }
$$

However, these physical methods suffer systematically from restrictions in the maximum detectable

\footnotetext{
'Author to whom correspondece should be addressed
}

interfacial areas, geometrical limitations, maldistribution of the bubbles, bubble identification problems and an underestimation of the value of $d_{s}$ for nonspherical bubbles. Besides that, it is doubtful whether the hydrodynamics of the dispersion are unaffected by the use of probes. Therefore, in our view the chemical method is still the best available method for the determination of interfacial areas in gas-liquid contactors, because if it is applied carefully an overall value for the effective interfacial area is directly obtained.

The reaction between oxygen and aqueous sodium sulphite solutions catalyzed by cobaltous ions $\mathrm{Co}^{2+}$ has been widely used as the standard model system for the chemical method. However, there is still disagreement about the reaction kinetics and especially about the reaction order in oxygen. This is probably caused by the sensitivity of the reaction towards small impurities in the water, the catalyst or the sulphite salt [see the review of Linek and Vacek (1981)]. The main advantage of this model system is the possibility to change the reaction rate constant over a wide range by changing the $\mathrm{Co}^{2+}$ catalyst concentration. However, it is restricted to aqueous solutions; moreover, the non-coalescecing behaviour of this ionic system also limits its use [see Bartos and Satterfield (1986)].

The reaction between $\mathrm{CO}_{2}$ and aqueous alkanolamine solutions was first introduced by Danckwerts and Sharma (1966) as an alternative model system for the determination of mass transfer parameters. Later Sridharan and Sharma (1976) showed that the reaction between $\mathrm{CO}_{2}$ and alkanolamines in organic and viscous solutions can also be used for the same purpose. The reaction rate can be varied by choosing the amine type. In recent years alkanolamines have been used to determine mass transfer parameters in 
various reactor types [see, amongst others, Metha and Sharma (1971), Midoux et al. (1984), Oyevaar et al. (1988) and Versteeg and van Swaaij (1988)].

Advantages of the reaction between $\mathrm{CO}_{2}$ and alkanolamines are:

(1) the ability to meet the different regimes of the chemical method by choosing different amines and different concentrations of these amines,

(2) the possibility to work with both aqueous and organic and viscous solutions as well, and

(3) the certainty that the reaction order in $\mathrm{CO}_{2}$ is cqual to onc.

The main disadvantage of this alternative model system is the rather high solubility of $\mathrm{CO}_{2}$ compared to other gases, which generally results in high $\mathrm{CO}_{2}$ conversions.

Schumpe and Deckwer (1980) showed that the combination of high conversions of the reactant in the gas phase and of non-uniform bubble sizes in gas-liquid dispersions can lead to an underestimation of the interfacial area. This is a direct result from the use of an overall conversion over the reactor to calculate the interfacial area, while the conversion within a single bubble depends non-linearly on the specific area of that bubble. Besides that, the bubbles may have different residence times $\tau_{b}$. These two phenomena, the use of an overall conversion and the different residence times of the bubbles, lead to deviations of the chemically determined interfacial area $a_{\text {chem }}$ from the true geometric interfacial area $a_{\text {geo }}$; the deviations become larger the broader the distribution of $\tau_{b} / d_{b}$ and the higher the conversion of the reactant in the gas phase [see Schumpe and Deckwer (1980)].

In order to calculate $a_{\mathrm{chem}} / a_{\mathrm{geo}}$ as a function of the overall conversion of the reactant in the gas phase and the bubble size distribution we will modify the method of Schumpe and Deckwer (1980). Model calculations will be given for both mechanically agitated reactors and bubble columns. For the mechanically agitated reactor it will be assumed that the bubble size distribution is represented by the distribution of Bayens (1967), while for the bubble column the bubble size distribution will be described by a log-normal distribution as proposed by Akita and Yoshida (1974).

In extension to Schumpe and Deckwer (1980), who assumed plug flow behaviour for the gas phase, a constant residence time $\tau_{b}$ for all bubbles, a complete segregation of the gas phase and no interaction between the bubbles, we will take into account the effect of the gas phase backmixing as well as the effect of bubble coalescence on the deviation of $a_{\text {chem }}$ from $a_{\text {geo }}$. In this study we present calculation results for a series of extreme macromixing and intermediate micromixing conditions of the gas phase:

(1) The gas bubbles do not interact, remain completely segregated and all bubbles have the same residence time $\tau_{b}$. We will call this the plug flow case.
(2) The gas bubbles do not interact, remain completely segregated and the residence times of the individual gas bubbles are distributed according to the distribution found in a completely mixed tank. We will call this the complete-mixing case.

(3) The gas bubbles do interact by means of coalescence and breakup processes. We will consider the two extreme cases of the individual bubbles all having the same residence time as in plug flow or all having a residence time distribution (RTD) as in a completely mixed tank. We will call this the intermediate-micromixing case. The micromixing of the gas phase will be simulated by the model of interaction by exchange with the mean (IEM model).

Results will be given for zero-, first- and secondorder reactions in the gas phase reactant. It will be shown that, for practical conditions and a first-order reaction for the gas phase reactant, which holds for the reaction between $\mathrm{CO}_{2}$ and alkanolamines, micro- and macromixing will reduce the deviation of $a_{\mathrm{chem}}$ from $a_{\text {geo }}$ substantially in comparison to the results for the extreme plug flow conditions of Schumpe and Deckwer (1980). All our model calculations are performed for non-shrinking bubbles and for absorption of a diluted gas phase reactant.

\section{THEORY}

\section{Chemical method}

The chemical method is based on the theory of chemically enhanced absorption of a gas phase reactant $A$ into a liquid in which an irreversible reaction takes place with a reactant $B$ [see Sharma and Danckwerts (1970) and Westerterp et al. (1984)]. The general equation for the chemically enhanced mole flux $J_{A}$ of a component $A$ from the gas into the liquid phase, as derived by Danckwerts (1970) using the penetration theory, is

$$
J_{A}=\frac{k_{L} \sqrt{1+H a^{2}}\left(m c_{A, G}-\frac{c_{A, L}}{1+H a^{2}}\right)}{1+\frac{m k_{L} \sqrt{1+H a^{2}}}{k_{\mathrm{G}}}} .
$$

The Hatta number, $\mathrm{Ha}$, equals

$$
H a=\frac{\sqrt{\frac{2}{n+1} k_{n, p} c_{B, L}^{p}\left(m c_{A, G}\right)^{n-1} D_{A}}}{k_{L}}
$$

if the chemical reaction rate in the liquid is given by $R_{c}=k_{n, p}\left(c_{B, L}\right)^{p}\left(c_{A, L}\right)^{n}$. In case the reaction is fast enough to consume $A$ already completely in the film or if the bulk volume is much greater than the film volume, the bulk concentration of component $A$ in the liquid, $c_{A, L}$, equals zero. Together with the fact that in most gas-liquid reactors and for systems with moderate reaction rates and low gas solubilities no gas phase 
mass transfer limitation occurs, eq. (2) becomes

$$
J_{A}=k_{L} m c_{A, G} \sqrt{1+H a^{2}} .
$$

With no depletion of component $B$ in the liquid phase near the interface and $\mathrm{Ha}>2$ the pseudo $n$ th-order mole flux $J_{A}$ becomes equal to

$$
J_{A}=K_{n} c_{A, G}^{(n+1) / 2}
$$

with

$$
K_{n}=\left[\frac{2}{n+1} k_{n, p} c_{B, L}^{p} D_{A} m^{(n+1)}\right]^{1 / 2} .
$$

Knowing $K_{n}$ and the RTD of the gas phase the interfacial area in a gas-liquid contactor can be obtained by measuring the overall conversion of the gas phase reactant, because the absorption rate $\phi_{A}$ equals $J_{A} a V_{R}$. Values of $K_{n}$ are usually determined separately in a gas-liquid contactor with a known interfacial area, i.e. a stirred cell or a wetted wall reactor. The RTD of the gas phase is normally chosen on the base of data from the literature.

\section{Absorption from a single bubble}

Schumpe and Deckwer (1980) considered the absorption of a reactant $A$ from a single bubble into a liquid in which a fast reaction occurs of the $n$ th-order in $A$ and for which $J_{A}$ can be described by eq. (5). After integration of the mass balance for a single bubble with the assumptions of constant pressure, of constant bubble size and of isothermal conditions, these a uthors obtained relations for the conversion $\zeta_{A}$; these are presented in Table 1 . In these relations $\zeta_{A}$ is given for reaction orders equal to 0,1 and 2 , respectively, as a function of the Stanton number $S t_{n}$ and the fraction $y_{o}$ of $A$ in the bubble at the inlet of the contactor.

$\zeta_{A}$ in a single bubble according to these relations is plotted in Fig. 1 for all three reaction orders. The value of $y_{0}$ is taken to be equal to 0.21 , which is the oxygen fraction in air. It can be seen that $\zeta_{A}$ depends nonlinearly on $S t_{n}$ and thus also non-linearly on the interfacial area $a$, which is proportional to $1 / d_{b}$ [see eq. (1)]. Only for low $\zeta_{A}$ can the dependence on the $a$ be taken as being linear. However, for higher $\zeta_{A}$ one must be aware that $\zeta_{A}$ depends non-linearly on $a$. In

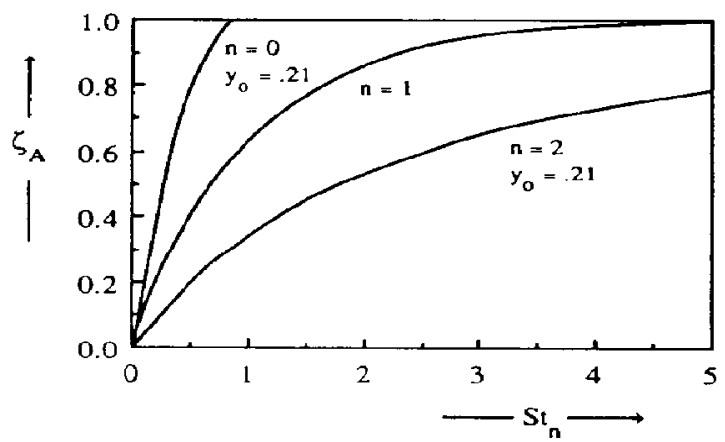

Fig. 1. Conversion of a reactant $A$ in the gas phase of a single bubble vs the Stanton number.

the case of different bubble sizes, as present in a gas-liquid dispersion, this non-linear dependence can give rise to considerable errors in the interfacial areas as calculated from the overall conversion of the gas phase reactant [see Schumpe and Deckwer (1980)].

\section{Absorption from a dispersion}

The bubble sizes in a gas-liquid dispersion are usually not uniform, but have a certain size distribution which depends on the operating conditions, the reactor geometry and the physico-chemical properties of the gas and the liquid phase. In order to calculate the effect of the bubble size distribution on the deviation of $a_{\text {chem }}$ from $a_{\text {geo }}$ it has to be known. For the mechanically agitated reactor we assumed that the bubble size distribution is represented by the distribution of Bayens (1967)

$$
E\left(\frac{d_{b}}{d_{s}}\right)=K \frac{1}{d_{s}}\left(\frac{d_{b}}{d_{s}}\right)^{2} \exp \left[-k^{2}\left(\frac{d_{b}}{d_{s}}\right)^{2}\right]
$$

with

$$
k=\frac{8}{3 \sqrt{\pi}} \text { and } K=\frac{4 k^{3}}{\sqrt{\pi}} .
$$

This distribution has been successfully used by Padmanabhan and Gal-Or (1968) and Midoux et al. (1980). For the bubble column the bubble diameters

Table 1. Relations for the conversion of a reactant $A$ in the gas phase of a single bubble with a fast reaction occurring in the liquid phase

\begin{tabular}{cccc}
$n$ & $\left.\left[\mathrm{~N} / \mathrm{m}^{2}\right)^{1 / 2(1-n)} \mathrm{K} / \mathrm{s}\right]$ & $S t_{n}$ & $\zeta_{A}$ \\
\hline 0 & $\sqrt{2 m k_{0, p} c_{B, L}^{p} D_{A}}$ & $K_{0} \frac{1}{\sqrt{P}} \frac{6 \tau_{b}}{d_{b}}$ & $1-\frac{\left(2 \sqrt{y_{o}}-S t_{o}\right)^{2}}{4 y_{o}}$ \\
1 & $m \sqrt{k_{1, p} c_{B, L}^{D} D_{A}}$ & $K_{1} \frac{6 \tau_{b}}{d_{b}}$ & $S t_{0} \leqslant 2 \sqrt{y_{0}}$ \\
2 & $m \sqrt{\frac{2}{3} m k_{2, p} c_{B, L}^{p} D_{A}}$ & $K_{2} \sqrt{P \frac{6 \tau_{b}}{d_{b}}}$ & $1-e^{-S t_{1}}$ \\
& & & $y_{o}\left(S t_{2}+\frac{2}{\sqrt{y_{o}}}\right)^{2}$
\end{tabular}




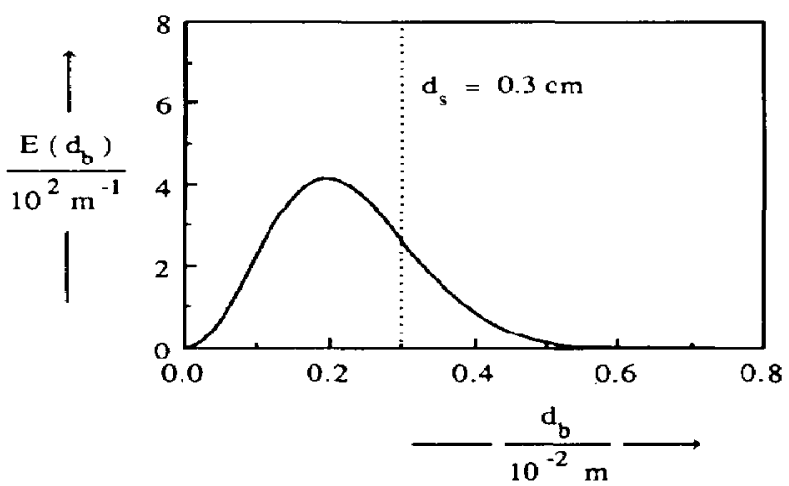

Fig. 2. Bayens distribution with $d_{s}=0.3 \mathrm{~cm}$.

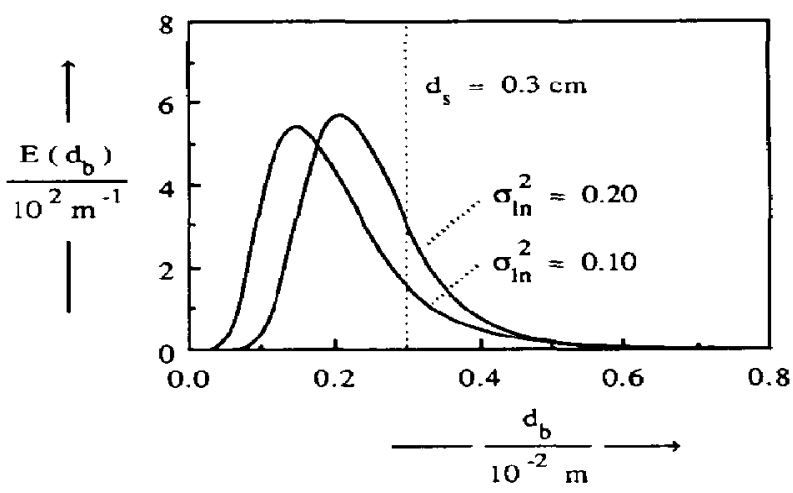

Fig. 3. Log-normal distribution with $d_{s}=0.3 \mathrm{~cm}$.

were assumed to follow a log-normal distribution:

$$
E\left(d_{b}\right)=\frac{1}{d_{b} \sigma_{l n} \sqrt{2 \pi}} \exp \left[-\frac{\left(\ln d_{b}-\ln d_{m b}\right)^{2}}{2 \sigma_{l n}^{2}}\right]
$$

which was proposed by Akita and Yoshida (1974) and used by Schumpe and Deckwer (1980) for their calculations. The two bubble size distribution functions are drawn in Figs 2 and 3 for a constant $d_{s}=3 \mathrm{~mm}$. According to experimentally determined data of Akita and Yoshida (1974) variances $\sigma_{i n}^{2}$ equal to 0.10 and 0.20 are realistic values for a log-normal distribution of bubble sizes in a bubble column.

Together with the assumption of complete segregation of the gas phase and thus no interaction between the bubbles, these distributions can be used for the calculation of the overall conversion $\Omega_{A}$ of the gas phase reactant $A$ in a gas-liquid contactor from

$$
\Omega_{A}=\frac{\int_{0}^{\infty} E\left(d_{b}\right) \zeta_{A}\left(d_{b}\right) d_{b}^{3} \mathrm{~d} d_{b}}{\int_{0}^{\infty} E\left(d_{b}\right) d_{b}^{3} \mathrm{~d} d_{b}}
$$

The conversion of reactant $A$ in a single bubble $\zeta_{A}\left(d_{b}\right)$ for eq. (9) can be obtained from the relations as given in Table 1 if the absorption rate constant $K_{n}$ and the residence time of the bubble, $\tau_{b}$, are known.

In an absorption experiment under these conditions an $\Omega_{A}$ equal to the one calculated from eq. (9) will be determined. The same values for $K_{n}$ and $z_{b}$ will also be used with the relations of Table 1 in order to evaluate the Stanton number $S t_{n \text {, chem }}$ and the effective interfacial area $a_{\text {chem }}$ from the experimentally determined $\Omega_{A}$. However, the relations between the overall conversion and the Stanton numbers as given in Table 1 are only valid for an ideally micromixed gas phase, and will therefore lead to deviations of $a_{\mathrm{chem}}$ from $a_{\mathrm{ges}}$. The assumed bubble diameter distributions can also be used to calculate the true geometrical values for $S t_{n, \text { geo }}$ and $a_{\text {geo }}$ from the Sauter mean bubble diameter. The values obtained for $a_{\text {chem }}$ and $a_{\text {seo }}$ can be compared and used to calculate the effects of the overall gas phase conversion and a bubble size distribution on the deviation of $a_{\mathrm{chem}}$ from $a_{\mathrm{geo}}$ under segregated conditions.

This deviation then represents the maximum error in the determination of the interfacial area by means of the chemical method. Any interaction between the bubbles will average the concentration of the gas phase reactant among the bubbles, and will therefore lead to smaller errors. In the next part of this study we will generalize this calculation model and evaluate the effects of both backmixing of the gas phase and coalescence of the bubbles on the deviations of $a_{\text {chem }}$ from $a_{\text {geo }}$ considerably.

\section{MODEL CALCULATIONS}

The plug flow case

Calculation hackgrounds. Schumpe and Deckwer (1980) showed that the error in $a_{\text {chem }}$ can be as large as $50 \%$ for fast gas-liquid reactions and high conversions of the reactant originally present in the gas phase. These authors assumed plug flow behaviour for the gas phase with a constant residence time $\tau_{b}$ for all bubbles and used the log-normal distribution as given in eq. (8) to calculate $a_{\text {chem }} / a_{\text {geo }}$ as a function of $\tau_{b}$. In their calculations they varied the variance $\sigma_{i n}^{2}$ and assumed the mean bubble diameter $d_{m b}$ to be constant. As a result of this assumption in their calculations $d_{s}$ and thus $a_{\text {geo }}$ varied with $\sigma_{l n}^{2}$. In our view it is better to keep $d_{s}$ constant in order to study the influence of $\sigma_{l n}^{2}$ on $a_{\text {shem }} / a_{\text {geo }}$, because only for this condition $a_{\text {geo }}$ is constant for each $d_{s}$. This will be done in the calculations that are presented in the next part of this study. $d_{m b}$ can then be calculated from the following relation as derived by Akita and Yoshida (1974) for the lognormal distribution function:

$$
\frac{d_{s}}{d_{m b}}=e^{2.5 \sigma_{i n}^{2}}
$$

Calculation results. Results on $a_{\text {chem }} / a_{\text {geo }}$, as calculated according to the aforementioned relations, under the assumption of plug flow behaviour for the gas phase with a constant residence time $\tau_{b}$ for all bubbles are given as a function of $\left(1-\Omega_{A}\right)$ in Fig. 4 . The results 


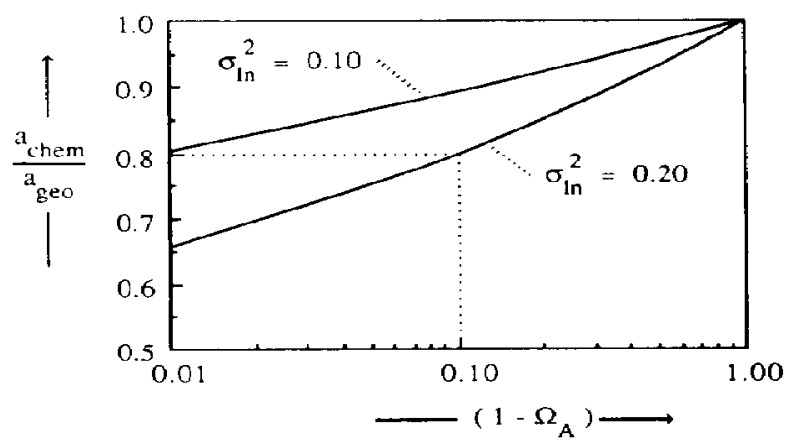

Fig. 4. Ratio $a_{\mathrm{chem}} / a_{\text {geo }}$ of a log-normal distribution with $d_{s}=3 \mathrm{~mm}$ vs $\left(1-\Omega_{A}\right)$ for a first-order reaction and plug flow of the gas phase.

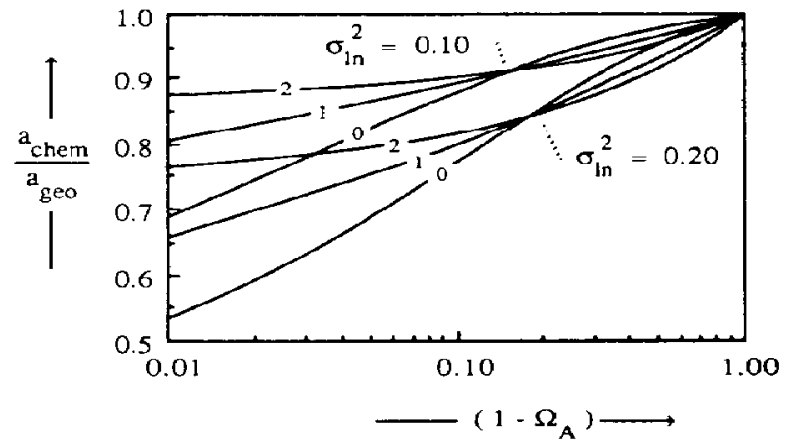

Fig. 5. Ratio $a_{\text {chem }} / a_{\text {geo }}$ of a log-normal distribution with $d_{s}=3 \mathrm{~mm}$ vs $\left(1-\Omega_{A}\right)$ for zero-, first- and second-order reactions and plug flow of the gas phase.

are obtained for a first-order reaction and a lognormal distribution with $d_{s}=3 \mathrm{~mm}$ and $\sigma_{i n}^{2}=0.10$ or 0.20 . It can be seen that errors in $a_{\text {chem }}$ larger than $20 \%$ occur only at $\Omega_{A}$ larger than 0.90 and that for $\Omega_{A}<0.99$ the errors in $a_{\text {chem }}$ will be always smaller than $35 \%$. Errors in the range of $50 \%$ as reported by Schumpe and Deckwer (1980) can only be obtained at extremely high $\Omega_{A}$ above 0.99 .

Plotting the results in the form of Fig. 4 revealed also that for a $\log$-normal distribution as well as for a Bayens distribution the ratio $\mathbf{a}_{\text {chem }} / \mathbf{a}_{\text {geo }}$ for zero-, firstand second-order reactions is only a function of $\boldsymbol{\Omega}_{A}$ and independent of $\mathrm{d}_{s}$ and the gas inlet fraction $\mathrm{y}_{0}$. This offers the possibility to generalize the results on $a_{\text {chem }} / a_{\text {geo }}$ independent from $d_{s}$ and $y_{o}$ in order to study the effects of bubble size distribution and $\Omega_{A}$ on the deviation of $a_{\text {chem }}$ from $a_{\mathrm{geo}}$ more closely.

Results as calculated on $a_{\text {chem }} / a_{\text {geo }}$ for all three reaction orders are plotted vs $\left(1-\Omega_{A}\right)$ in Fig. 5 for a log-normal distribution with $\sigma_{i n}^{2}=0.10$ and 0.20 . It can be seen that for $\Omega_{A}$ above 0.85 the deviation of $a_{\text {chem }}$ from $a_{\text {geo }}$ decreases with increasing reaction order.
The complete-mixing case

Calculation backgrounds. The assumptions of plug flow behaviour for the gas phase and of a constant residence time $\tau_{b}$ for all bubbles is certainly not realistic for a mechanically agitated gas-liquid reactor. The scarce literature data on the RTD of the gas phase for this reactor indicate that, above the critical agitation rate $N_{0}$ for a fully developed dispersion, the RTD of the gas phase is between that of one and two completely mixed tank reactors in series [see Hanhart et al. (1963) and Gal-Or and Resnick (1966)]. Therefore, it is justified as a first approximation to assume that the RTD of the gas phase in a mechanically agitated reactor is equal to that of a completely mixed tank reactor and can be described by the distribution function

$$
E(t)=\frac{1}{\tau} \exp \left(-\frac{t}{\tau}\right)
$$

If we furthermore assume that the bubbles of one particular diameter $d_{b}$ have the same RTD as the gas phase, we can calculate the overall conversion for each class of bubbles with diameter $d_{b}$ from

$$
\zeta_{A}\left(d_{b}\right)=\int_{0}^{\infty} E(t) \zeta_{A}\left(d_{b}, t\right) \mathrm{d} t
$$

For a zero- and first-order reaction eq. (12) can be solved analytically, which results for a zero-order reaction in

$$
\zeta_{A}\left(d_{b}\right)=\frac{S t_{o}}{\sqrt{y_{o}}}+\frac{S t_{o}^{z}}{2 y_{o}}\left[\exp \left(-\frac{2 \sqrt{y_{o}}}{S t_{0}}\right)-1\right]
$$

and for a first-order reaction in the well-known relation

$$
\zeta_{. A}\left(d_{b}\right)=\frac{S t_{1}}{1+S t_{1}} .
$$

For a second-order reaction eq. (12) can only be solved numerically. The conversion $\zeta_{A}\left(d_{b}\right)$ for a class of bubbles with a RTD of a completely mixed tank reactor according to these relations is plotted in Fig. 6 for all three reaction orders. After substitution of

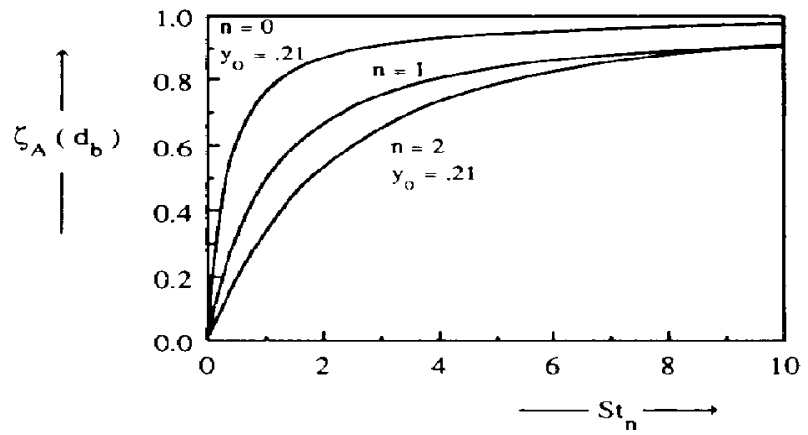

Fig. 6. Conversion of a reactant $A$ in a class of bubbles with a RTD of a completely mixed tank reactor vs the Stanton number. 
$\zeta_{A}\left(d_{b}\right)$ in eq. (9) $\Omega_{A}$ over the whole reactor of the gas phase reactant $A$ can be calculated. Especially for a second-order reaction these calculations demand long computation times.

The relations between $S t_{n, \text { chem }}$ and $\Omega_{A}$ for a completely mixed gas phase, which is perfectly mixed on a microscale, are different from the relations for plug flow as given in Table 1, because the driving force for mass transfer from the gas to the liquid phase is different. The following general relation between $S t_{n, \text { chem }}$ and $\Omega_{A}$ holds for a completely mixed gas phase, which is perfectly mixed on a microscale, and is used for the evaluation of absorption experiments:

$$
S t_{n, \text { chem }}=\frac{\Omega_{A}}{\left(1-\Omega_{A}\right)^{(n+1) / 2} y_{o}^{(n-1) / 2}} \text {. }
$$

These values for $S t_{n, \text { chem }}$ are then substituted in the relations for the Stanton numbers in Table 1 in order to evaluate the effective interfacial area $a_{\text {chem }}$.

For a completely mixed and segregated gas phase with only one uniform bubble diameter $d_{b} \boldsymbol{\Omega}_{A}$, as calculated from eq. (9), is equal to the experimentally determined conversion of the gas phase reactant. Only under these conditions $a_{\text {chem }}$ equals $a_{\text {geo }}$ at all $\Omega_{A}$. However, any non-uniformity in the bubble sizes will cause $a_{\text {chem }}$ to deviate from $a_{\text {geo }}$, because the conversion $\zeta_{A}\left(d_{b}\right)$ in a class of bubbles with diameter $d_{b}$ depends non-linearly on the interfacial area of the bubble (see Fig. 6).

Calculation results. We calculated $a_{\mathrm{chem}} / a_{\mathrm{geo}}$ for the two extreme RTDs of plug flow and of complete mixing under segregated conditions; the results are plotted in Fig. 7 for a Bayens distribution and for all three reaction orders. It can be concluded from Fig. 7

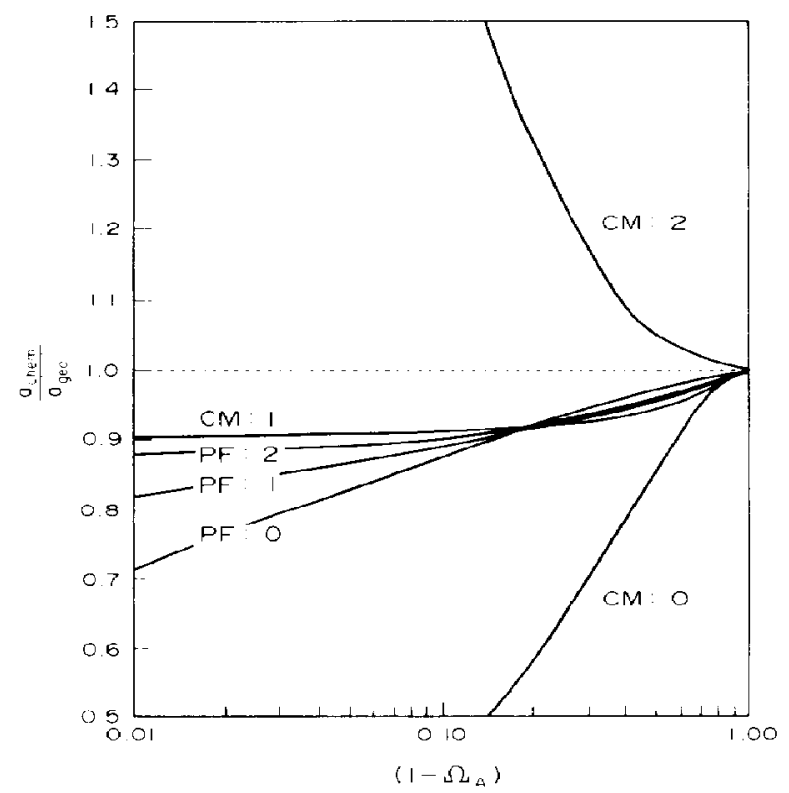

Fig. 7. Ratio $a_{\text {chem }} / a_{\text {geo }}$ of a Bayens distribution vs $\left(1-\Omega_{A}\right)$ for zero-, first- and second-order reactions and complete mixing (CM) or plug flow (PF) of the gas phase. that for a completely mixed gas phase and a first-order reaction the deviation of $a_{\mathrm{chem}}$ from $a_{\mathrm{geu}}$ will always be smaller than 0.90 for $\Omega_{A}<0.99$. However, for a zeroand second-order reaction the deviations of $a_{\mathrm{chem}}$ from $a_{\text {geo }}$ become considerably larger than for plug flow behaviour of the gas phase. For these two reaction orders the errors in $a_{\text {chem }}$ become larger than $50 \%$ for overall conversions $\boldsymbol{\Omega}_{\boldsymbol{A}}$ larger than 0.85 .

In the case of a zero-order reaction these large errors are caused by the presence of bubbles with $S t_{0}>2 y_{o}^{0.5}$ (see Table 1), which are depleted of gas phase reactant and do not contribute any more to the mass transfer from the gas to the liquid phase. This leads to an underestimation of the true interfacial area in the dispersion, because $a_{\text {chem }}$ is evaluated with the assumption that all bubbles contribute to the mass transfer until the gas phase is completely depleted of reactant.

The cascade model of $N$ completely mixed tank reactors in series can be used to describe intermediate macromixing behaviour of the gas phase in between plug flow and complete mixing. Some results for intermediate macromixing of the gas phase and a firstorder reaction are given in the Appendix. As expected these results are in between the results for the two extreme RTDs and do not alter the conclusions which can be drawn from Fig. 7.

The main conclusion now is that the error in $a_{\text {chem }}$, as determined by the chemical method, is affected considerably by the macromixing behaviour of the gas phase. For a zero- and second-order reaction and a completely mixed gas phase the error in $a_{\text {chem }}$ is a strong function of $\boldsymbol{\Omega}_{\boldsymbol{A}}$. Examples of gas-liquid model reaction systems with a zero and second order with respect to the gas phase reactant are the absorption of oxygen in aqueous dithionite and aqueous sulphite solutions, respectively [see Jhaveri and Sharma (1968) and Linek and Vacek (1981)]. We see that in a mechanically agitated gas-liquid reactor with a completely mixed gas phase the accuracy for these systems is always within $10 \%$ if $\Omega_{A}$ is kept below 0.40 and 0.60 , respectively, whatever the real micromixing behaviour of the gas phase will be. The use of these systems at higher conversion levels leads to extremely large errors in the determination of the interfacial area.

As mentioned before the major disadvantage of the reaction between $\mathrm{CO}_{2}$ and alkanolamine solutions is the rather high solubility of $\mathrm{CO}_{2}$, which can lead to high conversions of $\mathrm{CO}_{2}$. However, for the particular case of a mechanically agitated gas-liquid reactor and a first-order reaction, which is the case for the reaction between $\mathrm{CO}_{2}$ and alkanolamines, it can be concluded that the error in $a_{\text {enem }}$ will always be smaller than $10 \%$ for $\Omega_{A}$ smaller than 0.99 , provided the gas phase and each class of bubbles are completely mixed, whatever the real micromixing behaviour of the gas phase will be. This means that the gas-liquid model reaction systems with absorption of $\mathrm{CO}_{2}$ in alkanolamine systems can be used with a good accuracy for the determination of interfacial areas by the chemical method in mechanically agitated gas-liquid reactors. 


\section{The intermediate-micromixing case}

Calculation backgrounds. Up till now we have considered only the influence of the macromixing behaviour of the gas phase on the error in $a_{\text {chem }}$, while we assumed the gas phase to be completely segregated with, thus, no interaction between the gas bubbles. Therefore, we calculated the maximimum error in $a_{\text {chem }}$ if micromixing effects are totally neglected. However, especially in bubble columns with long residence times for the bubbles and in mechanically agitated gas-liquid reactors with high degrees of turbulence, the gas bubbles are likely to interact. The gas bubbles are subjected to complex coalescence and breakup processes on a microscale, which are unfortunately not very well understood. Qualitatively it can be argued that such interactions between the bubbles will level out the concentration differences of the gas phase reactant in the bubbles, and will therefore lead to a smaller error in $a_{\text {chem }}$.

In order to get an idea of the magnitude of the effect of bubble coalescence and breakup upon the deviation of $a_{\text {chem }}$ from $a_{\text {geo }}$ we adopted the well-known micromixing IEM model [see Villermaux (1981) and Westerterp et al. (1984)]. Villermaux (1981) calculated that the IEM nodel compares very well with the random coalescence model ( $R C . M$ ) in which a fluid is considered to contain only aggregates. These aggregates coalesce with each other and, after each coalescence, the two aggregates immediately split again into two new aggregates having the average composition of the two original aggregates. The whole process can be described by the coalescence frequency $\omega$, the number of coalescences per unit of time. This parameter has a clear physical meaning in a gas-liquid dispersion. However, this RCM requires complicated numerical techniques.

We therefore used the IEM model, which is easicr to compute and compares very well with the RCM as the characteristic micromixing parameter of the IEM model, $t_{m}$, equals $4 / \omega$ [see Villermaux (1981)]. In the IEM model a single agglomerate, which exchanges mass with the average content of the rest of the reactor, is followed from its birth at the reactor feed inlet. The average content is derived from the RTD for the reactor and from the concentration in typical agglomerates with different residence times.

The IEM model can be used to simulate the effects of bubble coalescence and breakup if the gas bubbles are considered as the agglomerates. For reasons of simplicity the IEM model will only be considered for a first-order reaction with respect to the gas phase reactant. The mass balance for the concentration of reactant $\mathbf{A}$ in a single bubble with diameter $d_{b}$ then equals

$$
\frac{\mathrm{d} c_{A}\left(d_{\mathrm{b}}, t\right)}{\mathrm{d} t}=\frac{c_{A, a v}-c_{A}\left(d_{b}, t\right)}{t_{m}}-\frac{6}{d_{b}} K_{1} c_{A}\left(d_{b}, t\right)
$$

in which $t_{m}$ is the time constant for mass exchange between the bubble and the rest of the reactor, and $c_{A, \text { av }}$ the average concentration of $A$ in the gas phase.
For a completely mixed gas phase, in which each class of bubbles with diameter $d_{b}$ has the same RTD as the gas phase, the average concentration $c_{A \text { av }}$ can be calculated from

$$
c_{A, \mathrm{av}}\left(d_{b}\right)=\int_{0}^{\infty} c_{A}\left(d_{b}, t\right) \frac{1}{\tau} \exp \left(-\frac{t}{\tau}\right) \mathrm{d} t
$$

and

$$
c_{A, \mathrm{av}}=\frac{\int_{0}^{\infty} E\left(d_{b}\right) c_{\mathcal{A}, \mathrm{av}}\left(d_{b}\right) d_{b}^{3} \mathrm{~d} d_{b}}{\int_{0}^{\infty} E\left(d_{b}\right) d_{b}^{3} \mathrm{~d} d_{b}} .
$$

For plug flow behaviour of the gas phase the bubble only exchange mass with the average content of the bubbles with the same residence time and the concentration $c_{A, \text { av }}(t)$ can be obtained from

$$
c_{A, \text { av }}(t)=\frac{\int_{0}^{\infty} E\left(d_{b}\right) c_{A}\left(d_{b}, t\right) d_{b}^{3} \mathrm{~d} d_{b}}{\int_{0}^{\infty} E\left(d_{b}\right) d_{b}^{3} \mathrm{~d} d_{b}} .
$$

Both sets [eqs (16)-(18) and eqs (16)-(19)] require an iteration procedure in order to be solved. The resulting average concentration $c_{A \text {. ar }}$ can be used to calculate the overall conversion $\Omega_{A}$, which can be substituted in eq. (15) and the relations of Table 1 in order to obtain $a_{\text {chem. }}$. The solutions of eqs (16) and (17) for complete mixing and plug flow of the gas phase are presented in Table 2.

Calculation results. Literature data on coalescence frequencies of gas bubbles in gas-liquid contactors are very scarce. For a mechanically agitated gas-liquid reactor Hassan and Robinson (1980) and Reith and Beek (1970) reported coalescence frequencies ranging from 1 to $15 \mathrm{~s}^{-1}$; the magnitude depending both on the operating conditions, like the superficial gas velocity and agitation rate, and on the liquid phase properties. We calculated with the IEM model the effect of coalescence on the deviation of $a_{\mathrm{chem}} / a_{\mathrm{geo}}$ for a completely mixed gas phase in a mechanically agitated reactor. The results are plotted in Fig. 8 for a Bayens distribution with $d_{s}=3 \mathrm{~mm}$, and for values of $t_{m}$ equal to $0.25,1$ and $4 \mathrm{~s}$, which correspond to coalescence frequencies of 16,4 and $1 \mathrm{~s}^{-1}$, respectively. The line representing a completely mixed gas phase under segregated conditions is also plotted in Fig. 8 . It can be seen that, as expected, coalescence of the gas bubbles reduces the deviation of $a_{\mathrm{chem}}$ from $a_{\mathrm{geo}}$. At the lowest time constant for mass exchange $t_{m}$ the error in a $a_{\text {chem }}$ becomes smaller than $5 \%$ for $\Omega_{A}$ lower than 0.99 . The calculations also show a slight influence of the assumed $d_{s}$ on the deviation of $a_{\text {chem }}$ from $a_{\mathrm{geo}}$.

To our knowledge no quantitative data on the coalescence frequencies of gas bubbles in a bubble column are available. However, from the papers of Calderbank (1967) and Deckwer et al. (1978) it can be qualitatively calculated that the coalescence frequencies of gas bubbles in a bubble column are in the 
Table 2. Relations for the fraction of reactant $A$ in a single bubble and for the average tank content for the IEM model with a fast reaction occurring in the liquid

$$
\begin{aligned}
& \text { Complete mixing } \\
& y_{A, a v}\left(d_{b}\right)=\frac{y_{A, \mathrm{av}}}{1+S t_{1}^{*} t_{m}} \frac{\frac{\tau}{t_{m}}+S t_{1}^{*} \tau}{1+\frac{\tau}{t_{m}}+S t_{1}^{*} \tau}+\frac{y_{A}\left(d_{b}, t=0\right)}{1+\frac{\tau}{t_{m}}+S t_{1}^{*} \tau} \\
& y_{A, a v}=\frac{\int_{0}^{\infty} E\left(d_{b}\right) y_{A, a v}\left(d_{b}\right) d_{b}^{3} \mathrm{~d} d_{b}}{\int_{0}^{\infty} E\left(d_{b}\right) d_{b}^{3} \mathrm{~d} d d_{b}}
\end{aligned}
$$

Plug flow

$$
\begin{aligned}
y_{A}\left(d_{b}, t\right) & =\frac{y_{A, \text { av }}(t)}{1+S t_{1}^{*} t_{m}}\left\{1-\exp \left[-\left(\frac{1}{t_{m}}+S t_{1}^{*}\right) t\right]\right\}+y_{A}\left(d_{b}, t=0\right) \exp \left[-\left(\frac{1}{t_{m}}+S t_{1}^{*}\right) t\right] \\
y_{A, \mathrm{av}}(t) & =\frac{\int_{0}^{\infty} E\left(d_{b}\right) y_{A}\left(d_{b}, t\right) d_{b}^{3} \mathrm{~d} d_{b}}{\int_{0}^{\infty} E\left(d_{b}\right) d_{b}^{3} \mathrm{~d} d_{b}}
\end{aligned}
$$

with $S t_{1}^{*}=\frac{S t_{1}}{\tau_{b}}$

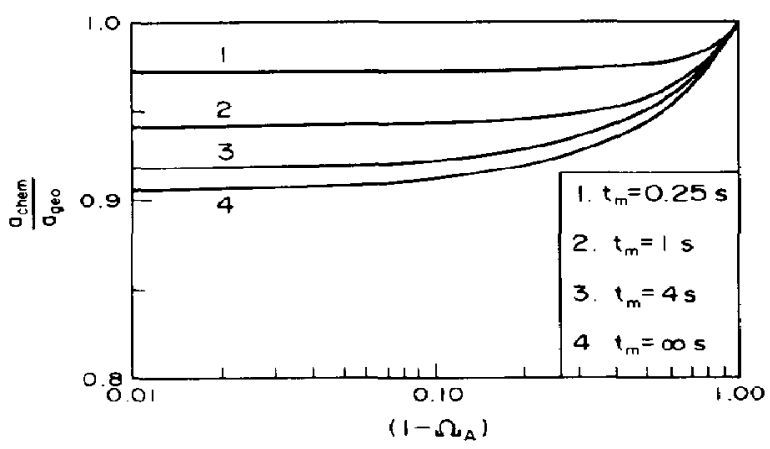

Fig. 8. Ratio $a_{\text {chem }} / a_{\text {geo }}$ of a Bayens distribution with $d_{\mathrm{s}}=3 \mathrm{~mm}$ vs $\left(1-\Omega_{A}\right)$ for a first-order reaction and complete mixing of the gas phase as calculated according to the IEM model.

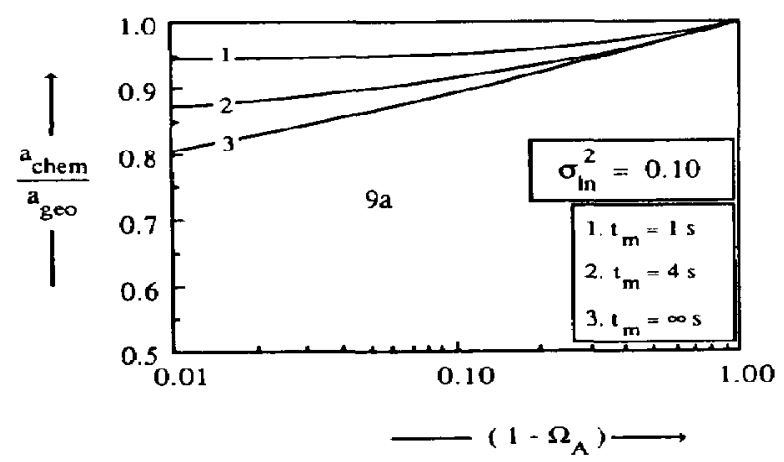

range of $0.5-5 \mathrm{~s}^{-1}$. Calculation results for a lognormal distribution in a bubble column with $\boldsymbol{d}_{s}$ $=5 \mathrm{~mm}$ with $\sigma_{l n}^{2}=0.10$ and 0.20 , and for values of $t_{m}$ equal to 1 and $4 \mathrm{~s}$, are plotted in Figs $9 a$ and $b$, respectively. The line representing plug flow behaviour of the gas phase under segregated conditions is given also. It can be seen that for these micromixing conditions the error in $a_{\mathrm{chem}}$ reduces considerably and is always smaller than $25 \%$ for $\Omega_{A}$ smaller than 0.99 . Also these calculations show a slight dependence of the results on $d_{s}$.

These calculations with the IEM model indicate that; if bubble coalescence and breakup in a dispersion of a gas-liquid contactor are taken into account, the deviation of $a_{\text {chem }}$ from $a_{\text {seo }}$, as compared to segregated conditions, becomes smaller. If realistic co-

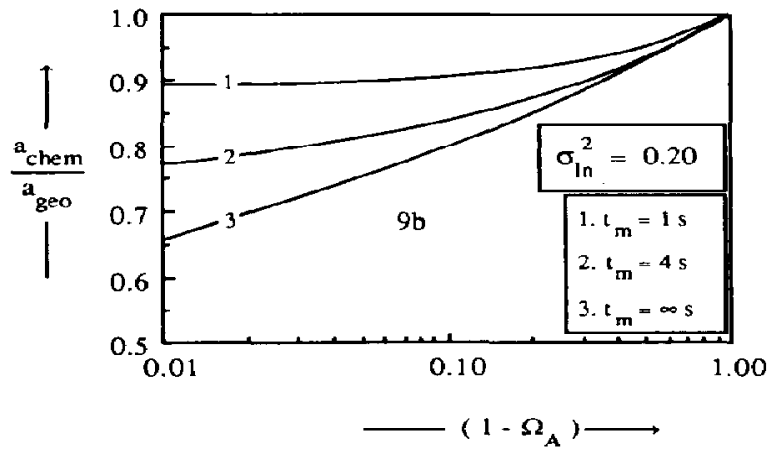

Fig. 9. Ratio $a_{\text {chem }} / a_{\text {geo }}$ of a log-normal distribution with $d_{s}=5 \mathrm{~mm}$ vs $\left(1-\Omega_{A}\right)$ for a first-order reaction and plug flow of the gas phase as calculated according to the IEM model. 
alescence frequencies are considered the error in $a_{\text {chem }}$ may reduce a factor 3 . For a mechanically agitated reactor and a first-order reaction with respect to the gas phase this means that the error in $a_{\text {chem }}$ becomes smaller than $5 \%$. For a bubble column and a firstorder reaction the calculations indicate that for most practical conditions the error in $a_{\text {chem }}$ is smaller than $20 \%$, if the overall conversion of the gas phase reactant, $\Omega_{A}$, is kept below 0.99 . The gas-liquid model reaction systems with absorption of $\mathrm{CO}_{2}$ in alkanolamine systems can therefore also be used with a rather good accuracy for the determination of interfacial areas by the chemical method in bubble column reactors.

\section{DISCUSSION AND CONCLUSIONS}

In this study it is shown that owing to the use of an overall conversion $\Omega_{A}$ for the gas phase reactant the evaluation of $a_{\text {chem }}$ from absorption experiments may lead to serious errors, because $\Omega_{A}$ represents an incorrect average if the bubble sizes and residence times are not uniform [see Schumpe and Deckwer (1980)]. These authors considered only plug flow behaviour for the gas phase under completely segregated conditions, while we studied for practical conditions the effect of gas phase backmixing as well as the effect of bubble coalescence and breakup on the deviation of $a_{\text {chem }}$ from $a_{\text {geo }}$. In several case studies we performed model calculations for both mechanically agitated gas-liquid reactors and bubble columns.

For a completely mixed gas phase in a mechanically agitated reactor it has been calculated that:

(1) For a first-order reaction with respect to the gas phase reactant and segregated conditions the error in $a_{\text {chem }}$ will always be smaller than $10 \%$ if $\Omega_{A}$ is lower than 0.99 .

(2) For a zero- and second-order reaction with respect to the gas phase reactant and segregated conditions the errors in $a_{\text {chem }}$ become larger than $50 \%$ for $\Omega_{A}$ larger than 0.85 and the errors in $a_{\text {chem }}$ will be smaller than $10 \%$ only for $\Omega_{A}<0.40$ and 0.60 , respectively.

(3) If bubble coalescence and breakup are taken into account, calculations for a first-order reaction according to the IEM model show that the error in $a_{\mathrm{chem}}$, compared to segregated conditions, reduces and becomes a factor 2-3 times smaller.

Therefore, it can be concluded that gas-liquid model reaction systems, which are zero- and secondorder in the gas phase reactant, result only in an accurate value for the interfacial area in a mechanically agitated gas-liquid reactor if $\Omega_{A}$ is low. Model reaction systems, which are first-order in the gas phase reactant, can be used very well for the determination of interfacial areas in a mechanically agitated reactor, provided the RTD of the gas phase is known. As already aforementioned the literature data on the RTD of the gas phase in this reactor are scarce. Hassan and Robinson (1980) and Oyevaar et al. (1988) showed that, for $\mathrm{CO}_{2}$ model reaction systems, which are first-order in $\mathrm{CO}_{2}$ and for which the overall conversions $\Omega_{A}$ is lower than 0.80 , the assumption of $a$ completely mixed gas phase leads to accurate values for the interfacial areas.

For plug flow behaviour of the gas phase in a bubble column it has been calculated that:

(1) For a first-order reaction and segregated conditions the error in $a_{\text {chem }}$ will always be smaller than $35 \%$ if $\Omega_{A}$ is lower than 0.99 .

(2) For segregated conditions the deviation of $a_{\mathrm{chem}}$ from $a_{\text {geo }}$ decreases with increasing reaction order for $\Omega_{A}$ above 0.85

(3) If bubble coalescence and breakup are taken into account, qualitative calculations for a first-order reaction according to the IEM model indicate that the error in $a_{\text {chem }}$, compared to segregated conditions, reduces and becomes smaller than $20 \%$ for $\Omega_{A}<0.99$.

These calculations demonstrate that the interfacial areas in a bubble column can be determined by the chemical method with a rather good accuracy even for overall conversions $\Omega_{A}$ up to 0.99 , provided the gas-liquid model reaction used is first-order in the gas phase reactant. The limits with respect to the overall conversion $\Omega_{A}$, as presented by Schumpe and Deckwer (1980) for a bubble column, are extended to higher values for $\Omega_{A}$, if bubble coalescence and breakup are taken into account. It should be kept in mind that in the calculations for the bubble column we considered the RTD of the gas phase to be plug flow. This will be the case at low superficial gas velocities in the bubbly flow regime and also in columns with a small diameter [see Shah et al. (1982)]. Deviations from plug flow will occur in the churn turbulent regime at higher superficial gas velocities and in columns with larger diameters due to liquid circulation and to different rising velocities of the bubbles.

It can be concluded that, provided the RTD of the gas phase is known, gas-liquid model reaction systems with absorption of $\mathrm{CO}_{2}$ in alkanolamine solutions can be used for the determination of accurate values for the interfacial areas in gas-liquid contactors, despite the high solubility of $\mathrm{CO}_{2}$ compared to other gases, which generally results in high $\mathrm{CO}_{2}$ conversions. Taking into account the advantages of the reaction between $\mathrm{CO}_{2}$ and alkanolamines compared to the traditional reaction between $\mathrm{O}_{2}$ and sodium sulphite as mentioned before, the use of $\mathrm{CO}_{2}$-alkanolamine systems for the determination of interfacial areas by the chemical method is therefore recommended.

Acknowledgements - The investigations were supported by the Netherlands Foundation for Chemical Research with financial aid from the Netherlands Organization for the Advancement of Scientific Research.

\section{NOTATION}

a specific interfacial area, $\mathrm{m}^{2} / \mathrm{m}^{3}$ dispersion c concentration, $\mathrm{mol} / \mathrm{m}^{3}$ 


\begin{tabular}{|c|c|}
\hline$d$ & diameter, m \\
\hline$d_{s}$ & Sauter mean bubble diameter, $m$ \\
\hline$d_{m b}$ & mean bubble diameter, $\mathrm{m}$ \\
\hline$D_{A}$ & $\begin{array}{l}\text { diffusivity of reactant } A \text { in the liquid phase, } \\
\mathrm{m}^{2} / \mathrm{s}\end{array}$ \\
\hline$E\left(d_{b}\right)$ & bubble diameter distribution function \\
\hline$E(t)$ & residence time distribution \\
\hline$E_{N}(t)$ & residence time distribution of a cascade \\
\hline $\mathrm{Ha}$ & Hatta number [eq. (3)] \\
\hline$J$ & mole flux, $\mathrm{mol} / \mathrm{m}^{2} \mathrm{~s}$ \\
\hline$k$ & mass transfer coefficient, $\mathrm{m} / \mathrm{s}$ \\
\hline$k_{m, p}$ & $\begin{array}{l}\text { reaction rate constant for a reaction of order } \\
(n, p), \mathrm{m}^{3(p+n-1)} / \mathrm{mol}^{(p+n-1)} \mathrm{s}\end{array}$ \\
\hline$K_{n}$ & absorption rate constant, $(\mathrm{N} / \mathrm{m})^{1 / 2(1-n)} \mathrm{m} / \mathrm{s}$ \\
\hline$m$ & distribution coefficient $\left(c_{L}=m c_{G}\right)$ \\
\hline$N$ & number of completely mixed tank reactors \\
\hline$N_{0}$ & critical agitation rate, $1 / \mathrm{s}$ \\
\hline$P$ & pressure, $\mathbf{P a}$ \\
\hline$R_{c}$ & chemical reaction rate, $\mathrm{mol} / \mathrm{m}^{3} \mathrm{~s}$ \\
\hline $\begin{array}{l}S_{t_{n}} \\
t\end{array}$ & $\begin{array}{l}\text { Stanton number for } n \text { th-order reaction } \\
\text { time, s }\end{array}$ \\
\hline$t_{m}$ & $\begin{array}{l}\text { time constant for mass exchange (IEM } \\
\text { model), } s\end{array}$ \\
\hline$v$ & $\begin{array}{l}\text { superficial velocity based on the empty } \\
\text { cross-sectional area of the vessel, } \mathrm{m} / \mathrm{s}\end{array}$ \\
\hline $\boldsymbol{V}$ & volume, $\mathrm{m}^{3}$ \\
\hline$y$ & molar fraction reactant in the gas phase \\
\hline \multicolumn{2}{|c|}{ Greek letters } \\
\hline$\varepsilon$ & hold-ul \\
\hline$\zeta$ & conversion \\
\hline$\sigma_{l n}^{2}$ & variance log-normal distribution \\
\hline$\tau$ & residence time, $s$ \\
\hline$\tau_{N}$ & $\begin{array}{l}\text { residence time in one tank reactor of cas- } \\
\text { cade, } s\end{array}$ \\
\hline$\phi$ & flow rate, $\mathrm{mol} / \mathrm{s}$ \\
\hline w & ncy, 1 \\
\hline$\Omega$ & overall conversion in gas-liquid contactor \\
\hline
\end{tabular}

\section{Subscripts}

$A$ reactant originally in the gas phase

av average content

$b$ bubble

$B$ reactant originally in the liquid phase

chem chemical

$G$ gas

geo geometric

$L \quad$ liquid

$n$ reaction rate order for reactant $A$ in the liquid phase

$o$ original

$p$ reaction rate order for reactant $B$ in the

$R \quad$ liquid $\mathrm{p}$

\section{REFERENCES}

Akita, K. and Yoshida, F., 1974, Bubble size, interfacial area and liquid phase mass transfer coefficients in bubble columns. Ind. Engng Chem. Process Des. Dev. 13, $84-91$.

Bayens, C.. 1967, Mass transfer in a dispersion. Ph.D. thesis, John Hopkins University Baltimore, MD.
Bartos, T. M. and Satterfield, C. N., 1986, Effects of finely divided solids on mass transfer between a gas and an organic liquid. A.I.Ch.E. J. 32, 773-781.

Buchholz, R., Zakrzewski, W. and Schügerl, K., 1979, Techniques for determining the properties of bubbles in bubble columns. Chemie-Ingr-Tech. 51, 568-575.

Calderbank, P. H., 1958, Physical rate processes in industrial fermentation. Part 2: the interfacial area in gas-liquid contacting with mechanical agitation. Trans. Instn chem. Engrs 36, 443-463.

Calderbank, P. H., 1967, Gas absorption from bubbles. Chem. Engr, Lond. 45, CE209 CE233.

Danckwerts, P. V. and Sharma, M. M., 1966, The absorption of carbon dioxide into solutions of alkalis and amines. Chem. Engr, Lond. 10, CE244-CE288.

Danckwerts, P. V., 1970, Gas-Liquid Reactions. McGrawHill, London.

Deckwer, W.-D., Adler, I. and Zaidi, A., 1978, A comprehensive study on $\mathrm{CO}_{2}$-interphase mass transfer in vertical cocurrent and countercurient gas-liquid flow. Can. J. chem. Engng 56, 43-55.

Gal-Or, B. and Resnick, W., 1966, Gas residence time in agitated gas-liquid contactor. Ind. Engng Chem. Des. Dev. 5, $15-19$.

Hanhart, J., Kramers, H. and Westerterp, K. R., 1963, The residence time distribution of the gas in an agitated gas-liquid reactor. Chem. Engng Sci. 18, 503-509.

Hassan, I. T. M. and Robinson, C. W., 1980, Mass-transfereffective bubble coalescence frequency and specific interfacial area in mechanically agitated gas-liquid contactor. Chem. Engng Sci. 35, 1277-1289.

Jhaveri, A. S. and Sharma, M. M., 1968, Absorption of oxygen in aqueous alkaline solutions of sodium dithionite. Chem. Engng Sci. 23, 1-8.

Linek, $V$. and Vacek, $V_{\text {., }} 1981$, Chemical engineering use of catalyzed sulphite oxidation kinetics for the determination of mass transfer characteristics of gas-liquid contactors. Chem. Engng $S_{c i .}$ 36, 1747 - 1768.

Mehta, V. D. and Sharma, M. M., 1971, Mass transfer in mechanically agitated contactors. Chem. Engng Sci. 26, $461-479$

Midoux, N., Laurent, A. and Charpentier, J. C., 1980, Limits of the chemical method for the determination of physical mass transfer parameters in mechanically agitated gas-liquid reactors. A.I.Ch.E. J. 26, 157-162.

Midoux, N., Morsi, B. I., Purwasasmita, M., Laurent, A. and Charpentier, J. C., 1984, Interfacial area and liquid side mass transfer coefficient in trickle bed reactors operating with organic liquids. Chem. Engng Sci. 39, 781-794.

Oyevaar, M. H., Zijl, A. D. and Westerterp, K. R., 1988, Interfacial areas and gas hold-ups at elevated pressures in a mechanically agitated gas-liquid reactor. Chem. Engng Technol. 11, 1-10.

Padmanabhan, L. and Gal-Or, B., 1968, Analysis of the effect of particle size and residence time distribution on heat and mass transfer with linear source in particulate systems. Chem. Engng Sci. 23, 631-643.

Reith, T. and Beek, W. J., 1968, Gas holdups, interfacial areas and mass transfer coefficients in gas-liquid contactors. Proc. 4th Eur. Symp. chem. Reaction Engng, Brussels, pp. 191-204.

Reith, T. and Beek, W. J., 1970, Bubble coalescence rates in a stirred tank contactor. Trans. Instn chem. Engrs 48, T63-T68.

Schumpe, A. and Deckwer, W.-D., 1980, Analysis of chemical methods for determination of interfacial areas in gas-liquid dispersions with non-uniform bubble sizes. Chem. Engrg Sci. 35, 2221-2233.

Schumpe, A. and Deckwer, W.-D., 1982, Comparison of the photographic and the sulphite oxidation method for interfacial area determination in bubble columns. Chem. Engng Commun. 17, 313-324.

Shah, Y. T., Kelkar, B. G., Godbole, S. R. and Deckwer, W.-D., 1982, Design parametcrs estimations for bubble column reactors. A.I.Ch.E. J. 28. 353-379. 
Sharma, M. M. and Danckwerts, P. V., 1970, Chemical methods of measuring interfacial area and mass transfer coefficients in two-fluid systems. Br. chem. Engng 15, $522-528$.

Sridhar, T. and Potter, O. E., 1978, Interfacial area measurements in gas-liquid agitated vessels. Chem. Engng Sci. 33, $1347-1353$.

Sridharan, K. and Sharma, M. M., 1976, New systems and methods for the measurement of effective interfacial area and mass transfer coefficients in gas-liquid contactors. Chem. Engng Sci. 31, 767-774.

Versteeg, G. F. and van Swaaij, W. P. M., 1988, Absorption of $\mathrm{CO}_{2}$ and $\mathrm{H}_{2} \mathrm{~S}$ in aqueous alkanolamine solutions using a fixed-bed reactor with cocurrent downfow operation in the pulse flow regime. Submitted for publication to Chem. Engng Proc.

Villermaux, J., 1981, Drop break-up and coalescence. Micromixing effects in liquid-liquid reactors, in Multiphase Chemical Reactors (Edited by Rodrigues, Sweet and Calo). Noordhof, Goroningen.

Westerterp, K. R., van Dierendonck, L. L. and de Kraa, J., 1963. Interfacial areas in agitated gas-liquid contactors. Chem. Engng Sci. 18, 157-176.

Westerterp, K. R., van Swaaij, W. P. M. and Beenackers, A. A. C. M., 1984, Chemical Reactor Design and Operation. John Wiley, New York.

\section{APPENDIX}

The distribution function of the cascade model with $N$ completely mixed tank reactors in series equals

$$
E_{N}(t)=\frac{N^{N} t^{(N-1)}}{\tau_{N}^{N}(N-1) !} \exp \left(-N \frac{t}{\tau_{N}}\right)
$$

and can be used in eq. (12) to calculate the conversion of the gas phase reactant for each class of bubbles $\zeta_{A}\left(d_{b}\right)$. For a firstorder reaction eq. (12) can be solved analytically and result

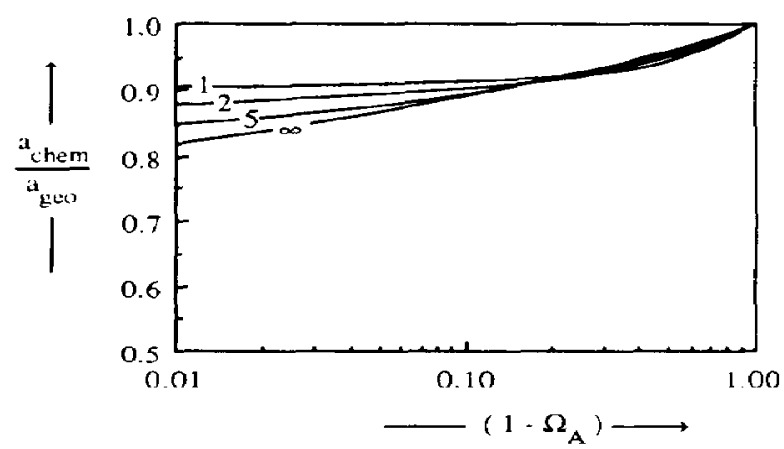

Fig. A1. Ratio $a_{\text {chem }} / a_{\text {geo }}$ of a Bayens distribution vs $\left(1-\Omega_{A}\right)$ for a first-order reaction and intermediate macromixing behaviour.

in the well-known relation

$$
\zeta_{A}\left(d_{b}\right)=1-\left(\frac{1}{1+S t_{1, N} \tau_{N}}\right)^{N} .
$$

$S t_{1 . \text { chem }}$ can be calculated from

$$
S t_{1, \text { chem }}=N\left[\frac{1}{\left(1-\overline{\left.\Omega_{A}\right)^{1 / N}}-1\right.}\right] \text {. }
$$

The limits of the cascade model represent plug flow bchaviour for $N \rightarrow \infty$ and complete mixing for $N=1$. Results on $a_{\text {chem }} / a_{\text {geo }}$ for those two extreme RTDs were already shown in Fig. 6 and are plotted again in Fig. A1 together with results for a cascade of two and five completely mixed tank reactors in series for a first-order reaction. As expected it can be seen that the lines, which represent the intermediate macromixing behaviour, lie in between the lines for the two extreme RTDs. 